\title{
Experimental investigations of monolithic IGBT transistor acoustic emission phenomena
}

\author{
Maciej Kozak ${ }^{1}$, Radosław Gordon ${ }^{1, *}$ \\ ${ }^{1}$ Maritime University of Szczecin, Faculty of Marine Engineering, 70-500 Szczecin, Poland
}

\begin{abstract}
Many recent researches are focused on the diagnostic methods allowing determination of the condition of working semiconductor components (power transistors, diodes etc.). As any solid material under stresses (mechanical, temperature and electrical) the semiconductors are generating elastic waves in the case of changing conductivity state. Based on the results of experimental research, a correlation can be observed between the transition between the on and off states of a single IGBT transistor and the emitted acoustic signal. Acquisition of acoustic emission signals was obtained with use of a wide band acoustic emission sensor. The received signal initially was prepared by $\mathrm{AD}$ input card with amplifier and then were recorded by means of a computer software. Acquired data were exported the to a file, which enabled further processing of the measured signals. The aim of the study was to determine the acoustic emission background signal coming from switching IGBT transistor and preparation of frequency response. The results of analysis will be used as a reference point for a further investigation of early-stage faults of semiconductors elements.
\end{abstract}

\section{Introduction}

\subsection{Goal and object of the study}

One of the most wanted feature of modern power electronics devices and power electronic systems operating in high-reliability systems is the possibility of early-stage failure detection. Taking care of the quality of equipment and its maintenance, an important factor is their diagnostics and the earliest possible detection of damages occurring in the modules and parts. The ideal case would be to anticipate the failure of a device with enough time to take remedial action.

Therefore, development and creation of a diagnostic system for power electronic devices, based on acoustic emission sensors has begun. As the first object of research IGBT monolithic transistors were chosen [1].

\subsection{Examples of IGBT transistor applications}

The IGBT transistors are meant for operation of medium frequency power electronics circuits and are capable of high voltages and currents switching, which results in a significant power dissipation in their volume. Due to the nature of medium and high frequency switching circuits only two states of operation of the IGBT transistor are considered for measurement and testing of acoustic emission signals.

The insulated gate bipolar transistors are most often used in inverter drive systems, e.g. to control the rotational speed of squirrel-cage motors [2] but also in grid side inverter systems [4]. The variety of applications and recent interest in the researches of the subject matter [3] in the context of bipolar power transistors use points out the importance of the presented issue.

\subsection{Structure of the IGBT transistor}

The IGBT transistor combines the advantages of low switching voltage (MOSFET) and high current and voltage (BJT) conductivity [1]. The modular construction of the IGBT transistor causes the formation of additional capacities and parasitic inductance, which during charging are responsible for the shape of the acoustic wave [5]. Another factor responsible for the acoustic waves generation are temperature changes inside the silicon structure caused by current flow across the connectors and supply connections [6].

\section{The measurement layout}

The IRG4BC40S transistor was put under experiments on the testbench. The WS Alfa acoustic emission sensor was attached to the case of the tested transistor. Before performing the experimental tests, the system was calibrated in accordance to the manufacturer's recommendations [7].

The signal was amplified by a wide band amplifier, which output is connected to a 24-bit A/D converter and for recording AudaCity software has been chosen.

\footnotetext{
Corresponding author: R.Gordon@AM.Szczecin.pl
} 
AudaCity was converting the recorded signal to a WAV format file. The WAV file was then processed and analyzed in Matlab software.

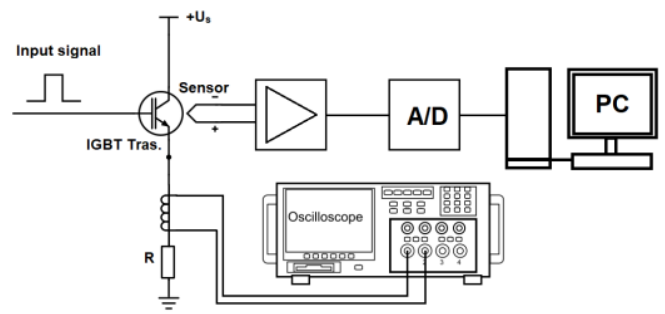

Fig. 1. Diagram of a measured system for testing acoustic emission phenomena in an IGBT transistor.

The transistor switching signals came signal generator. The collector of the tested transistor was loaded with a high-power resistor, and the current through the power supply system, transistor and resistor to ground was measured with an oscilloscope.

\section{Measure and results}

The possibility of observing the phenomena of acoustic emission in modules with transistors has been proved in earlier publications [2], [8].

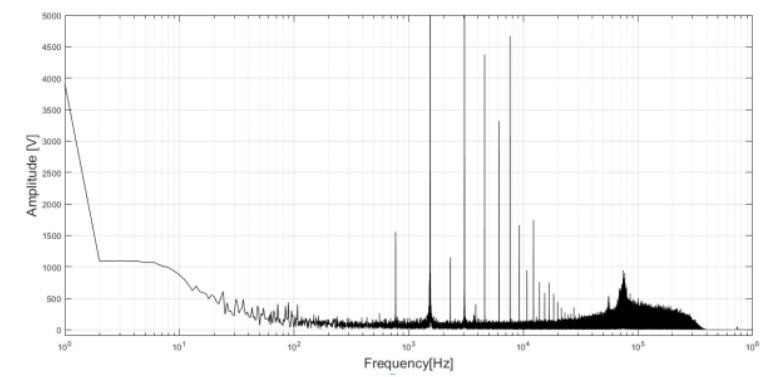

Fig. 2. Frequency spectrum of the saved signal (own study)

The goal of investigations was to amplify, receive and digitize signal in time domain and save it on the computer to a WAV files. The obtained data were imported to Matlab for further processing. With the Matlab processing the time range was narrowed down and enlarged, which allowed to record the switching moment of the IGBT transistor.

The extinguishing waveform can be the effect of impulse reflections from different nodes inside the structure of the transistor. Comparing this graph with other cases of acoustic emission, the curve obtained in the time domain can be described by one of the equations representing the acoustic emission wave.

Since the signal itself in the time domain carries little information for the observer, the next step was to transform the signal into another form. The most commonly used transformation is FFT.

The height of the spectral bands corresponds to the amplitude of the sinusoidal components. Since the acoustic emission signal had a higher amplitude than the interference, in this case it is easy to identify it and determine in what frequency range the transistor switching emission is included. Figure 2 shows the FFT spectrum of the recorded signal.
This manual narrowing of the band can be compared to the operation of a band filter. With an appropriate algorithm, any frequency band can be distinguished. Such a filter can be realized digitally by processing the signal e.g. in Matlab. After cutting off not needed frequencies coming from internal waves reflections, the signal can be further processed using other transformers or return to the time domain with the noisy signal in the time domain.

\section{Conclusions}

The paper presents the results of measurements carried out on a switching single semiconductor element. However, the origin of the signal (e.g. thermal changes in the housing, changes in the shape of the semiconductor) was not examined. The emission source will be tested in separate tests. The best result for further tests would be to note changes in the structure of the semiconductor at the current flow. The researches included also other switching frequencies and variable voltages applied to collector-emitter. The aim of this procedure was to find a switching signal pattern and characteristic frequencies of a correctly working transistor. Signals. acquired during tests are the basis for next researches conducted on faulty IGBT's.

\section{References}

1. B.J. Baliga, The IGBT Device Physics, Design and Applications of the Insulated Gate Bipolar Transistor, Elsevier Inc., (2015).

2. R. Gordon, Influence of chosen parameters on electrical machines bearings exploitation. $58^{\text {th }}$ International Conference of Machine Design Departments - ICMD 2017, Prague, 94-99, (2017).

3. T. Karkkainen,. J. Talviti, M. Kuisma, J. Hannonen, J. Strom, Acoustic Emission in Power Semiconductor Modules-First Observations, IEEE transactions on power electronics, 29, (2014).

4. M. Kozak, Initial Excitation Issues of Synchronous Generator with VSI Inverter in Varying Rotational Speed Operation, MAPE, 1, (2018).

5. A. Bejger, M. Kozak, Acoustic Emission Of Monolithic IGBT Transistors. Symso (2017).

6. B. Nagl, B. Czerny, M. Lederer, G. Khatibi, Experimental investigation of transient electrical, thermal and mechanical behavior of IGBT inverter modules during operation, ISPSD, Japan (2013).

7. J. Keprt, P. Benes, Determination of Uncertainty in Calibration of Acoustic Emission Sensors, $4^{\text {th }}$ International Conference on NDT, Chania (2007).

8. R. Gordon, A. Dreas, Detection Of Acoustic Emission In Semiconductors, ISBN 978-83-6526525-8, MAPE 2018. 